\title{
Temporal and Spatial Variations in the Twinning Rate in Norway
}

\author{
Johan Fellman \\ Hanken School of Economics, Helsinki, Finland
}

\begin{abstract}
Strong geographical variations have been noted in the twinning rate (TWR). In general, the rate is high among people of African origin, intermediate among Europeans, and low among most Asiatic populations. In Europe, there tends to be a south-north cline, with a progressive increase in the TWR from south to north and a minimum around the Basque provinces. The highest TWRs in Europe have been found among the Nordic populations. Furthermore, within larger populations, small isolated subpopulations have been identified to have extreme, mainly high, TWRs. In the study of the temporal variation of the TWR in Norway, we consider the period from 1900 to 2014. The regional variation of the TWR in Norway is analyzed for the different counties for two periods, 1916-1926 and 1960-1988. Heterogeneity between the regional TWRs in Norway during 1916-1926 was found, but the goodness of fit for the alternative spatial models was only slight. The optimal regression model for the TWR in Norway has the longitude and its square as regressors. According to this model, the spatial variation is distributed in a west-east direction. For 19601988, no significant regional variation was observed. One may expect that the environmental and genetic differences between the counties in Norway have disappeared and that the regional TWRs have converged towards a common low level.
\end{abstract}

- Keywords: assisted reproductive technology, geographical co-ordinates, multicollinearity, regression analysis, county

Strong geographical variations have been observed in the twinning rate (TWR). The TWR is high among people of African origin, intermediate among Europeans, and low among most Asiatic populations (Eriksson, 1973). In Europe, there tends to be a progressive increase in the TWR from south to north, with a minimum around the Basque provinces on the border between Spain and France. The highest TWRs in Europe have been noted among the Nordic populations (Bulmer, 1970; Eriksson, 1964, 1973; James 1985). Furthermore, within larger populations some small isolated subpopulations have been identified to have extreme, mainly high, TWRs.

Fellman and Eriksson (1990) examined the regional variation in the TWR in Finland for 1974-1983. Eriksson et al. (1993) presented a detailed study of the secular changes in the Nordic countries of Denmark, Finland, and Sweden. In our studies of the regional variation of the TWR in Sweden, we have analyzed TWRs for the different counties (Eriksson \& Fellman, 2004; Fellman \& Eriksson, 2003, 2004, 2005a, 2009). In Fellman (2016), the temporal variation in the Norwegian TWR was compared with corresponding trends in the neighboring Nordic countries of Iceland and Denmark.

\section{Material and Methods}

\section{Materials}

To evaluate the temporal variations in the TWR in Norway, we consider TWRs for 1900-2014 (Table 1). A deep trough can be found in 1960-1989. After 1990, a marked increase in the TWR is noted that cannot be explained by the slight increase in mean maternal age. The main cause of the recovery of the TWR is noted to be the influence of assisted reproductive technologies (ART) and particularly the use of fertility-enhancing drugs on the commonly noted dependence between maternal age and TWR (Fellman \& Eriksson, 2005a). Therefore, model building of normal TWRs should be based on data obtained before 1960 .

The study of the spatial variation in the TWR is based on data grouped according to the Norwegian counties for 1916-1926. The available data are presented in Table 2. We

RECEIVED 15 February 2016; ACCEPTED 31 March 2016. First published online 24 June 2016.

AdDress fOr CORRESPONDENCE: Professor Johan Fellman, Hanken School of Economics, Helsinki, Finland. E-mail: fellman@hanken.fi 


\section{TABLE 1}

Temporal Variation in the Twinning Rate in Norway (1900-2014)

\begin{tabular}{lccr}
\hline Period & Maternities & Twin maternities & TWR \\
\hline 1900-1909 & 641,958 & 8,659 & 13.49 \\
$1910-1919$ & 619,280 & 8,688 & 14.03 \\
$1920-1929$ & 574,871 & 8,377 & 14.57 \\
$1930-1939$ & 447,160 & 6,211 & 13.89 \\
$1940-1949$ & 599,246 & 7,583 & 12.65 \\
$1950-1959$ & 629,647 & 7,766 & 12.33 \\
$1960-1969$ & 651,663 & 6,894 & 10.58 \\
$1970-1979$ & 578,655 & 5,469 & 9.45 \\
$1980-1989$ & 525,020 & 5,372 & 10.23 \\
$1990-1999$ & 593,781 & 8,601 & 14.49 \\
$2000-2009$ & 572,335 & 10,330 & 18.05 \\
$2010-2014$ & 295,946 & 4,826 & 16.31 \\
Total & $6,729,562$ & 88,776 & 13.19 \\
\hline
\end{tabular}

\section{TABLE 2}

Regional Twinning Rates in Norway (1916-1926) Grouped According to the Norwegian Counties (or Fylkes)

\begin{tabular}{lcllll}
\hline Total & Maternities & TWR & Latitude & Longitude & $\overline{T W R}$ \\
\hline Östfold & 40,599 & 14.1875 & 59.2833 & 11.2000 & 15.13 \\
Akershus & 92,584 & 14.0953 & 59.9333 & 10.7500 & 15.06 \\
Hedmark & 43,196 & 15.7304 & 60.7833 & 11.0500 & 15.11 \\
Oppland & 38,423 & 15.6937 & 61.1167 & 10.4167 & 15.00 \\
Buskerud & 31,625 & 14.5771 & 59.7333 & 10.1500 & 14.95 \\
Vestfold & 30,550 & 15.9411 & 59.2833 & 10.4167 & 15.00 \\
Telemark & 31,524 & 15.0044 & 59.2000 & 9.5500 & 14.84 \\
Aust-Agder & 17,058 & 14.7442 & 58.4667 & 8.7667 & 14.67 \\
Vest-Agder & 26,706 & 15.1092 & 58.1667 & 8.0000 & 14.49 \\
Rogaland & 38,287 & 12.3802 & 58.9500 & 5.7167 & 13.85 \\
Hordaland & 62,970 & 12.7125 & 60.3667 & 5.4000 & 13.75 \\
Sogn og Fjordane & 21,640 & 15.7344 & 61.2167 & 6.7833 & 14.19 \\
Möre & 41,548 & 15.7771 & 62.7500 & 7.2333 & 14.29 \\
Sör-Tröndelag & 43,436 & 14.1935 & 63.4167 & 10.3833 & 15.00 \\
Nord-Tröndelag & 23,075 & 15.0813 & 64.0500 & 11.7167 & 15.21 \\
Nordland & 49,318 & 14.3658 & 67.3000 & 14.5333 & 15.48 \\
Troms & 25,637 & 16.1487 & 69.6667 & 18.9333 & 15.44 \\
Finnmark & 15,091 & 12.9216 & 70.0667 & 29.7333 & 12.96 \\
Total & 673,266 & 14.544 & 61.8750 & 11.1519 & \\
\hline Note The & & & & & \\
\hline
\end{tabular}

Note: The latitudes and longitudes are the coordinates of the residences of the counties. The TWR is the observed TWR of the county, and $\overline{T W R}$ is the estimated TWR of the county according to model (5). For details, see the text.

have considered a period for which the normal TWR rates are maximal. One may expect that the environmental and genetic differences have later disappeared and that the regional TWRs have converged towards a common low level. This may have influenced the TWR for 1960-1988.

\section{Methods}

We applied different spatial regression models to the regional TWRs. The map of Norway and its counties is presented in Figure 1. Following Fellman and Eriksson (2009), the location of the counties is defined as the geographic coordinates of the corresponding residences. The coordinates for Norway are eastern longitude and northern latitude. The coordinates of the counties (residences) in Norway are also given in Table 2.

\section{Multicollinearity}

We analyzed the spatial variation in the TWR with alternative regression models. The regressand is the TWR in the different counties and the presumptive regressors are the longitude (meridian) $M$, and the latitude $L$, and the regressors of second order, $M^{2}, L^{2}$, and $L M$. The regressors $M$ and $L$ are defined as deviations from their national cluster means, and consequently, the intercepts obtained are the estimated TWRs in the center of the cluster.

The elongated or drawn-out format of Norway and the inclusion of the whole set of regressors, $M, M^{2}, L, L^{2}$, and $L M$, indicate that attention must be paid to the multicollinearity between the regressors. Concerning regional studies of Swedish twins, this was also observed by Fellman and Eriksson (2009). The multicollinearity pattern can show marked variations. Therefore, different measures based on the eigenvalues of the correlation matrix have been proposed in the literature. In the review of the literature, Fellman (1981) and Fellman and Eriksson (2009) have given detailed presentations and analyses of different measures of multicollinearity. In the following, a short presentation is given.

Consider a set of variables (regressors) $u_{1}, u_{2}, \ldots, u_{n}$ and their correlation matrix

$$
\boldsymbol{C}=\left[\begin{array}{cccccc}
1 & c_{12} & \ldots & c_{1 j} & \ldots & c_{1 n} \\
c_{21} & 1 & \ldots & c_{2 j} & \ldots & c_{2 n} \\
\cdot & . & . & . & . & \cdot \\
c_{i 1} & c_{i 2} & \ldots & c_{i j} & \ldots & c_{i n} \\
\cdot & \cdot & . & \cdot & . & . \\
c_{n 1} & c_{n 2} & \ldots & c_{n j} & \ldots & 1
\end{array}\right]
$$

where $c_{i j}=\operatorname{cor}\left(u_{i}, u_{j}\right)$. If the variables are mutually uncorrelated the correlation matrix equals the identity matrix

$$
\boldsymbol{I}=\left[\begin{array}{cccccc}
1 & 0 & \ldots & 0 & \ldots & 0 \\
0 & 1 & \ldots & 0 & \ldots & 0 \\
. & . & . & . & . & . \\
0 & 0 & \ldots & 1 & \ldots & 0 \\
. & . & . & . & . & . \\
0 & 0 & \ldots & 0 & \ldots & 1
\end{array}\right] .
$$

The eigenvalues are obtained in the following way: Solve the equation

$$
\begin{aligned}
\operatorname{det}(\boldsymbol{C}-\lambda \boldsymbol{I}) & =\operatorname{det}\left[\begin{array}{cccccc}
1-\lambda & c_{12} & \ldots & c_{1 j} & \ldots & c_{1 n} \\
c_{21} & 1-\lambda & \ldots & c_{2 j} & \ldots & c_{2 n} \\
\cdot & \cdot & . & \cdot & . & \cdot \\
c_{i 1} & c_{i 2} & \ldots & c_{i j} & \ldots & c_{i n} \\
\cdot & \cdot & . & \cdot & . & \cdot \\
c_{n 1} & c_{n 2} & \ldots & c_{n j} & \ldots & 1-\lambda
\end{array}\right] \\
& =0 .
\end{aligned}
$$

This equation is an algebraic equation of degree $n$. Consequently, it has $n$ roots $\lambda_{1}, \lambda_{2}, \ldots, \lambda_{n}$, which are the eigenvalues of the matrix $C$. For every correlation matrix, the roots are real and non-negative and $\sum_{i=1}^{n} \lambda_{i}=n$. We number the roots such that $0 \leq \lambda_{1} \leq \lambda_{2} \leq \ldots \leq \lambda_{n}$. If the variables are uncorrelated, the correlation matrix is a unit matrix and all the eigenvalues equal one. If there are marked 


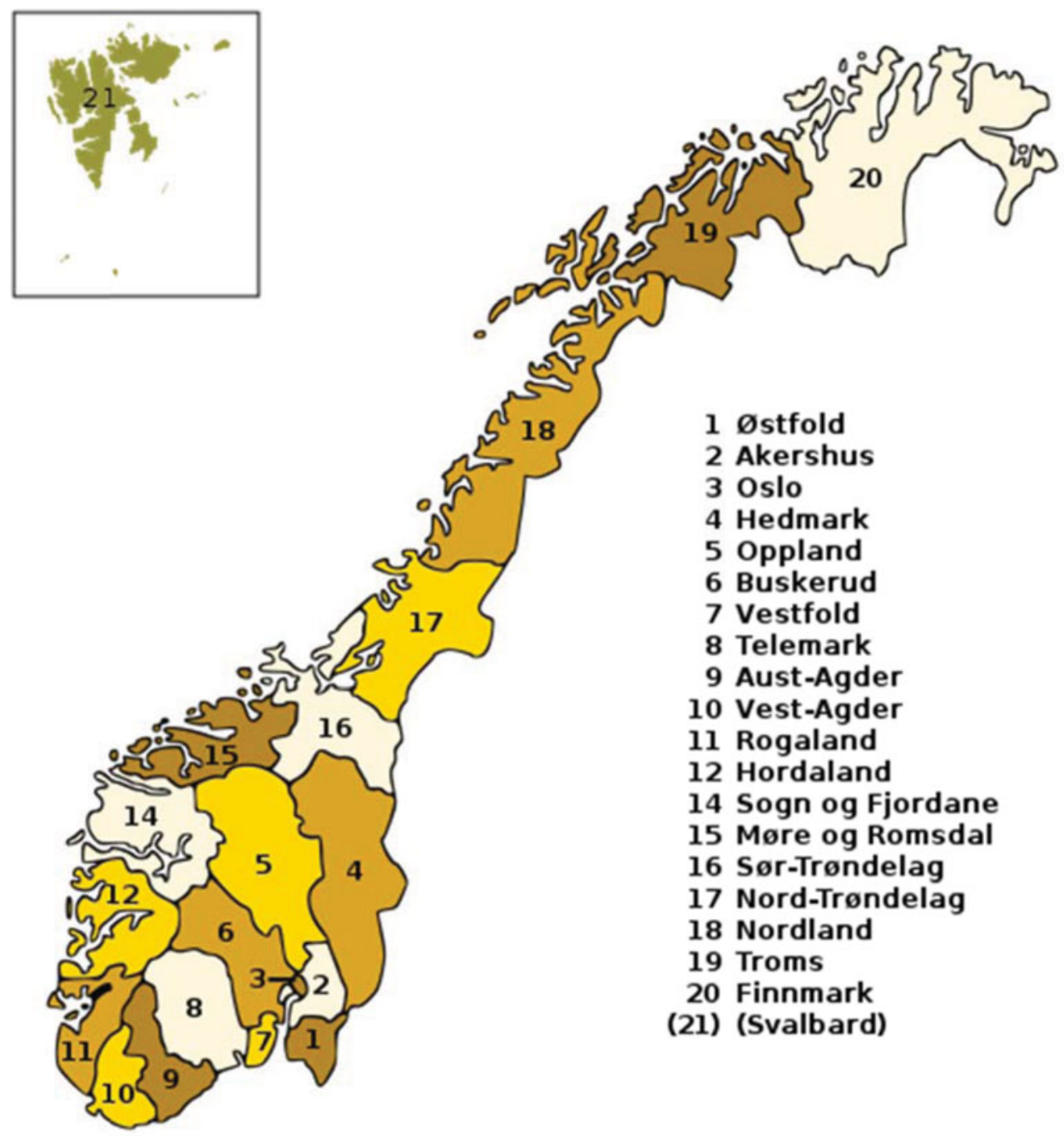

FIGURE 1

(Colour online) Map of Norway including the counties.

correlations between the variables, one speaks about multicollinearity and there are small eigenvalues. The multicollinearity causes reduced accuracy in the estimates. If $\lambda_{1}=0$, then at least one exact linear relation between the regressors can be found and the correlation matrix is singular and not all parameters are estimable.

Fellman (1981) discussed the pros and cons of the different multicollinearity measures. He stated that a good measure should satisfy the following conditions: (a) the measure defines a critical level above (or below) which the corresponding correlation matrix should be considered strongly multicollinear; (b) the measure can be used for comparisons between different correlation matrices.

These properties imply that the measure must be (at least in a loose sense) 'monotonic' and the effect of the dimension of the matrix on the measure should not be too great. The measures of multicollinearity were also discussed in Fellman and Eriksson (2009).
In Table 3, we present some commonly used measures of collinearity and their basic properties. The simplest measure is the inverse of the minimum eigenvalue $m_{1}=\lambda_{1}^{-1}$. In the uncorrelated case, $m_{1}=1$, but with increasing multicollinearity $\lambda_{1} \rightarrow 0$ and $m_{1}$ increases towards infinity.

The next measure, proposed by Wichern and Churchill (1978) and Casella (1980), is $m_{2}=\frac{\lambda_{n}}{\lambda_{1}}$. This is defined as the condition number of the matrix. In the uncorrelated case, $m_{2}=1$ and with increasing multicollinearity $m_{2}$ increases towards infinity.

The determinant of the correlation matrix, $m_{3}=$ $\operatorname{det}(C)=\lambda_{1} \lambda_{2} \cdots \lambda_{n}$, has also been used as a measure of multicollinearity. In the uncorrelated case, $m_{3}=1$, but with increasing multicollinearity $m_{3}$ decreases towards zero.

Mahajan et al. (1977) and Lawless (1978) considered the sum $m_{4}=\sum \frac{1}{\lambda_{i}}$. For uncorrelated variables, its value is $n$. With increasing multicollinearity, it increases towards infinity. 


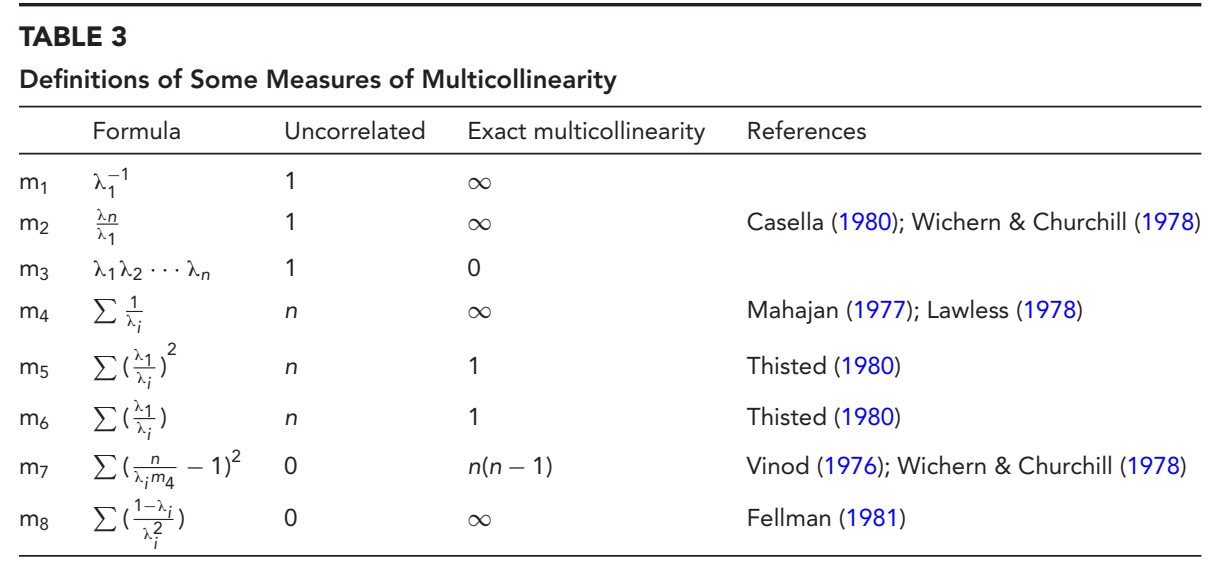

Note: The measures $m_{3}, m_{4}$ and $m_{8}$ were chosen for this study. For details, see the text.

Thisted (1980) suggested two measures, $m_{5}=\sum\left(\frac{\lambda_{1}}{\lambda_{i}}\right)^{2}$ and $m_{6}=\sum\left(\frac{\lambda_{1}}{\lambda_{i}}\right)$. These measures satisfy the inequalities $1<m_{5} \leq m_{6} \leq n$. The equality signs hold only in the orthogonal case. For uncorrelated variables, these measures obtain the value $n$, and when the multicollinearity increases they converge towards one.

The measure $m_{7}=\sum\left(\frac{n}{\lambda_{i} m_{4}}-1\right)^{2}$ was introduced by Vinod (1976). It is zero for complete orthogonal systems, but according to Vinod the components in the sum will be large for non-orthogonal data. However, the value of $m_{7}$ depends greatly on the relative proportions between the eigenvalues and does not satisfy the assumption of a monotone function.

The measure $m_{8}=\sum\left(\frac{1-\lambda_{i}}{\lambda_{i}^{2}}\right)$ was introduced by Fellman (1981), who presented arguments for its suitability as a multicollinearity measure and proved that $m_{8} \geq 0$, with equality in the orthogonal case, and that $\lim _{\lambda_{1} \rightarrow 0} m_{8}=\infty$.

The measures $m_{1}$ and $m_{2}$ are simple to handle, but their weakness is that they are mainly based on the smallest eigenvalue. Hence, any other small eigenvalues are almost ignored. The measure $m_{3}$ depends strongly on the dimension of the matrix and is suitable only for matrices with low dimension. The advantage of $m_{4}$ over $m_{1}$ and $m_{2}$ is that it takes into account the effect of several small eigenvalues. In addition, one can show that mathematically it has an evident connection with the estimation problem. Thisted (1980) recommended $m_{5}$ in estimation and $m_{6}$ in prediction situations. The main criticism against these measures is that they can be used if there is one extremely small eigenvalue, but if there are several small eigenvalues the measure is rather worthless. The measure $m_{7}$ is useful only if we are dealing with a correlation matrix with only one small eigenvalue.

When we consider the variables $M, M^{2}, L, L^{2}$, and $L M$, the dimension is low and several small eigenvalues may exist. Consequently, $m_{3}, m_{4}$, and $m_{8}$ could be recommended and used in this study.

\section{Results}

\section{Temporal Trends}

The temporal variation in the TWR in Norway (1900-2014) is presented in Figure 2. The TWR shows strong fluctuations. One observes that the TWR is rather constant until the 1950s, but there is a maximum in the 1910s and 1920s. There is a marked trough in the 1970s. After that, there is an increase up to the maximum 18.05 per 1,000 in $2000-$ 2009. The main cause of this recovery of the TWR seems to be the influence of ART (Fellman \& Eriksson, 2005a). A slight decrease in the TWR can be observed after 2009. Such recaptures can also be observed in other studies and are explained by improved treatment techniques in order to avoid multiple maternities. For sake of comparison, the TWR for Denmark is included in Figure 2. The temporal trends for the TWRs in both countries are similar. The temporal trend of the seasonality in the TWR in Norway is discussed in Fellman and Eriksson (1999). More detailed studies of the TWR and general demographic studies of Denmark can be found in Eriksson and Fellman (1999); Fellman and Eriksson (2005a); and Fellman (2015).

\section{Regional Variation}

The data during the periods 1916-1926 and 1960-1988 were used in the study of the regional heterogeneity, and these data are indicated in Figure 2. In Table 2, we have presented the TWR distributed over the Norwegian counties for 1916-1926. When we applied a $\chi^{2}$ test of the regional variation for the period 1916-1926, we obtained $\chi^{2}=56.8$ with 17 degrees of freedom and $p<.001$, indicating statistically significant variations. Below, we follow Fellman and Eriksson (2009) and build spatial models of the regional variations.

We controlled as a check the regional TWRs for 19601988 (Table 4) and for this period found no significant regional variations $\left(\chi^{2}=21.59\right.$ with 18 degrees of freedom, $p>.05)$. Our results indicate that for these two periods 


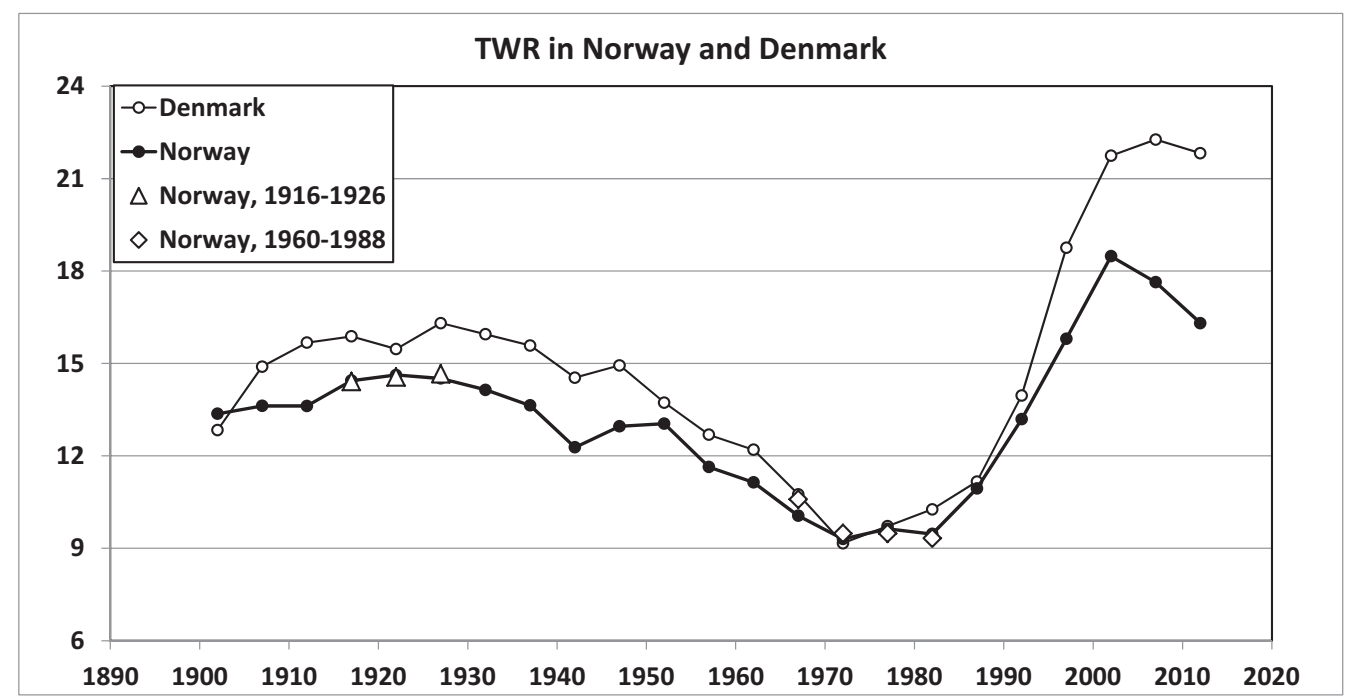

\section{FIGURE 2}

Temporal variation in the twinning rate in Norway (1900-2014). Note: The $\Delta$ symbol indicates data analyzed in the regional study. The $\diamond$ symbol indicates late regional data. For comparison sake, the TWR for Denmark is included in the figure. The temporal trends for the TWRs in both countries are similar.

TABLE 4

Regional Data in Norway for the Period 1960-1988.

\begin{tabular}{lrrrc}
\hline County & Maternities & Latitude & Longitude & \multicolumn{1}{c}{ TWR } \\
\hline Östfold & 85,929 & 59.2833 & 11.2000 & 9.356562 \\
Akershus & 80,239 & 59.9333 & 10.7500 & 9.745884 \\
Oslo & 212,837 & 59.9333 & 10.7500 & 10.20969 \\
Hedmark & 86,420 & 60.7833 & 11.0500 & 9.939829 \\
Oppland & 64,963 & 61.1167 & 10.4167 & 10.02109 \\
Buskerud & 75,724 & 59.7333 & 10.1500 & 9.996831 \\
Vestfold & 70,435 & 59.2833 & 10.4167 & 9.576205 \\
Telemark & 61,609 & 59.2000 & 9.5500 & 9.803762 \\
Aust-Agder & 36,920 & 58.4667 & 8.7667 & 9.507042 \\
Vest-Agder & 56,394 & 58.1667 & 8.0000 & 10.76356 \\
Rogaland & 135,336 & 58.9500 & 5.7167 & 9.871727 \\
Hordaland & 171,890 & 60.3667 & 5.4000 & 9.482809 \\
Sogn og Fjordane & 54,726 & 61.2167 & 6.7833 & 9.757702 \\
Möre og Romsdal & 97,585 & 62.7500 & 7.2333 & 9.90931 \\
Sör-Tröndelag & 102,912 & 63.4167 & 10.3833 & 9.4838 \\
Nord-Tröndelag & 56,646 & 64.0500 & 11.7167 & 9.515235 \\
Nordland & 104,007 & 67.3000 & 14.5333 & 10.15316 \\
Troms & 70,643 & 69.6667 & 18.9333 & 9.979757 \\
Finnmark & 41,273 & 70.0667 & 29.7333 & 8.795096 \\
Total & $1,666,488$ & 61.7728 & 11.1307 & 9.822151 \\
\hline
\end{tabular}

spatial models can only be applied in the data set for 19161926. These findings can be compared with the results obtained by Fellman and Eriksson (2005b) that the regional TWRs for Sweden converged from 1750 to 1960 towards a common low level.

The elongated/drawn-out format of Norway suggests that we consider the multicollinearity in the regional models for Norway. We calculated the correlation coefficients between the TWR and the regressors $M, M^{2}, L, L^{2}$, and $L M$. The correlation matrix is given in Table 5 . For the correlation matrix of the regressors, we obtain $\lambda_{1}=0.00175, \lambda_{2}=$ $0.11089, \lambda_{3}=0.15308, \lambda_{4}=0.44883$, and $\lambda_{5}=4.28945$.
One eigenvalue is extremely small and at least two can be considered rather small.

The multicollinearity measures for our Norwegian data are presented in Table 6 and a strong multicollinearity is obvious. Below, we define the optimal model consisted of the regressors $M$ and $M^{2}$ that we accept as optimal. For the regressors in this model, the multicollinearity is markedly reduced. Now, the two eigenvalues are $\lambda_{1}=0.168015$ and $\lambda_{2}=1.831985$. The corresponding multicollinearity measures for this reduced model are also included in Table 6. A comparison of the values of $m_{3}, m_{4}$, and $m_{8}$ shows how much stronger the multicollinearity is for the larger model. Moving from the large model to the small, $m_{3}$ increases from 0.0000566 to 0.307801 , and $m_{4}$ and $m_{8}$ decrease from 590.4 to 6.50 and from 327166 to 29.2 , respectively. Table 6 includes the Swedish data given by Fellman and Eriksson (2009). One observes that the multicollinearity in the Norwegian data is markedly stronger than in the Swedish data.

\section{Regression Models}

First we build the linear regression model for the total set of regressors. The obtained model is:

$$
\begin{aligned}
\text { TWR }= & 15.007+0.15197 M+0.00975 L+0.00090 M^{2} \\
& +0.03401 L^{2}-0.04957 L M .
\end{aligned}
$$

The goodness of fit of the model, measured with the adjusted coefficient of determination, is $\bar{R}^{2}=0.080$, indicating a bad fit. This is supported by the low model test value $F=1.298$. None of the parameter estimates is statistically significant.

When we tried to improve the regional variation with different combinations of north and south trends, no model 


\section{TABLE 5}

Correlation Coefficients Between TWR and Regressors for the Norwegian Data

\begin{tabular}{lllllll}
\hline Correlation matrix & TWR & L & M & LL & MM & LM \\
\hline TWR & 1.00000 & 0.00264 & -0.09512 & -0.12832 & -0.39806 & -0.32385 \\
L & 0.00264 & 1.00000 & 0.80700 & 0.80700 & 0.62600 & 0.71500 \\
M & -0.09512 & 0.80700 & 1.00000 & 0.87900 & 0.83200 & 0.89600 \\
LL & -0.12832 & 0.80700 & 0.87900 & 1.00000 & 0.77100 & 0.90200 \\
MM & -0.39806 & 0.62600 & 0.83200 & 0.77100 & 1.00000 & 0.96800 \\
LM & -0.32385 & 0.71500 & 0.89600 & 0.90200 & 0.96800 & 1.00000 \\
\hline
\end{tabular}

Note: A strong multicollinearity can be identified among the regressors.

TABLE 6

Multicollinearity Measures $m_{3}, m_{4}$ and $m_{8}$ for Our Norwegian Data

\begin{tabular}{lllcc}
\hline & Norway & Norway optimal & Sweden & Sweden, optimal \\
& $M, M^{2}, L, L^{2}, L M$ & $M, M^{2}$ & $M, M^{2}, L, L^{2}, L M$ & $M, M^{2}, L, L^{2}$ \\
\hline $\mathrm{m}_{3}$ & 0.0000566 & 0.307801 & 0.015 & 0.159 \\
$\mathrm{~m}_{4}$ & 590.4 & 6.50 & 25.336 & 8.904 \\
$\mathrm{~m}_{8}$ & 327166.0 & 29.2 & 288.347 & 19.600 \\
\hline
\end{tabular}

Note: One can observe that the multicollinearity for the optimal model is markedly reduced and can be ignored. As a comparison, we include Swedish data presented in Fellman and Eriksson (2009). The Norwegian data show markedly stronger multicollinearity.

gave a good fit, but all abridged models reduced the multicollinearity. The best model obtained was a linear and a quadratic west-east model that contains the regressors Mand $M^{2}$. The estimated model is:

$$
\text { TWR }=15.12362+0.15482 M-0.01461 M^{2} .
$$

The $M^{2}$ parameter is significant and the $M$ parameter is almost significant. The standard errors are $S E_{\hat{\alpha}}=$ $0.280353, S E_{\hat{\beta}_{M}}=0.076354$, and $S E_{\hat{\beta}_{M^{2}}}=0.005334$. The adjusted coefficient of determination is $\bar{R}^{2}=0.251$ and the test statistics $F=3.855$. Hence, the model is markedly better than Equation (4), but the goodness of fit for Equation (5) is only slight. However, this model having a westeast trend has to be accepted as the optimal model. This model indicates the tendency of a central maximum for $M=5.298$, and the value decreases in both western and eastern directions (cf. Figure 3). In fact, attempts to improve the model by including $M$-terms of higher degree were quite fruitless.

We have explained the TWR with the geographical coordinates, and consequently, the pattern of the level curves is simple. If we assume that model (5) holds, we can then obtain parabolic level curves for the TWR. Let $T W R_{0}=R$ be a constant value, then the equation of the corresponding level curve is $\beta_{M} M+\beta_{M^{2}} M^{2}+\alpha-R=0$, indicating a parabola with a vertical axis and the opening to the south. Furthermore, the axis obtained for the longitude is independent of the chosen TWR level. According to the parameter estimates, the axis corresponds to the longitude $=$ $16.45^{\circ}$ E. Summing up, we consider Equation (5) as the best model, and the TWR estimates obtained are included in Table 2 and presented in Figure 4. For $R=0$ the level curve generates model (5).

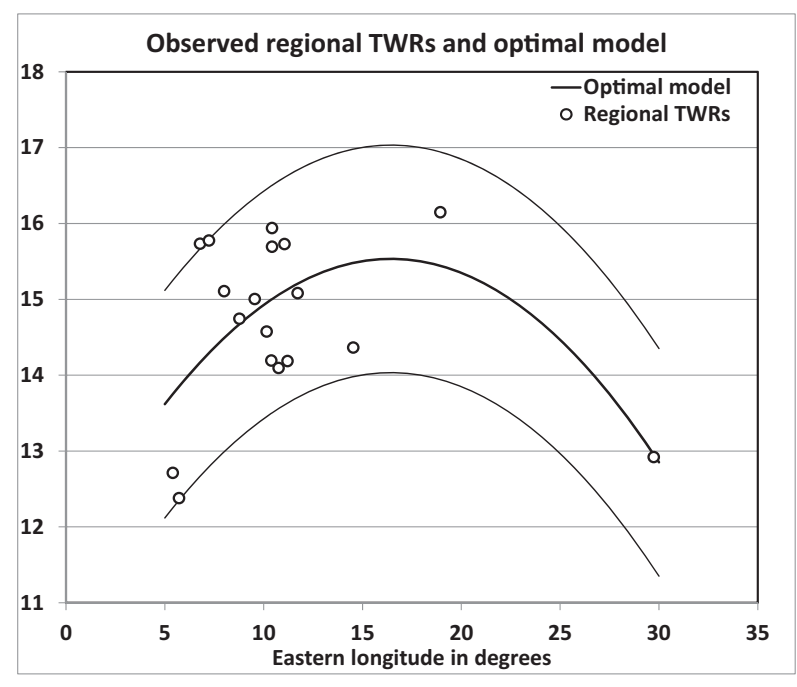

\section{FIGURE 3}

Associations between regional TWRs (1916-1926) and the optimal model. Note: A slight east-western effect of second degree can be found. This model indicates the tendency of a central maximum and decreases in both western and eastern directions. The TWRs for the central counties show values divergent from the model. This finding explains the slight goodness of fit. Attempts to improve the model by including $M$-terms of higher degree were quite fruitless. Two level curves are included in the figure.

\section{Discussion}

The analyses of the spatial models for Norway indicate that horizontal trends have to be considered. Counties with low TWR values can be found in the western counties of Hordaland and Rogaland and in the north-eastern county of Troms. The central counties 


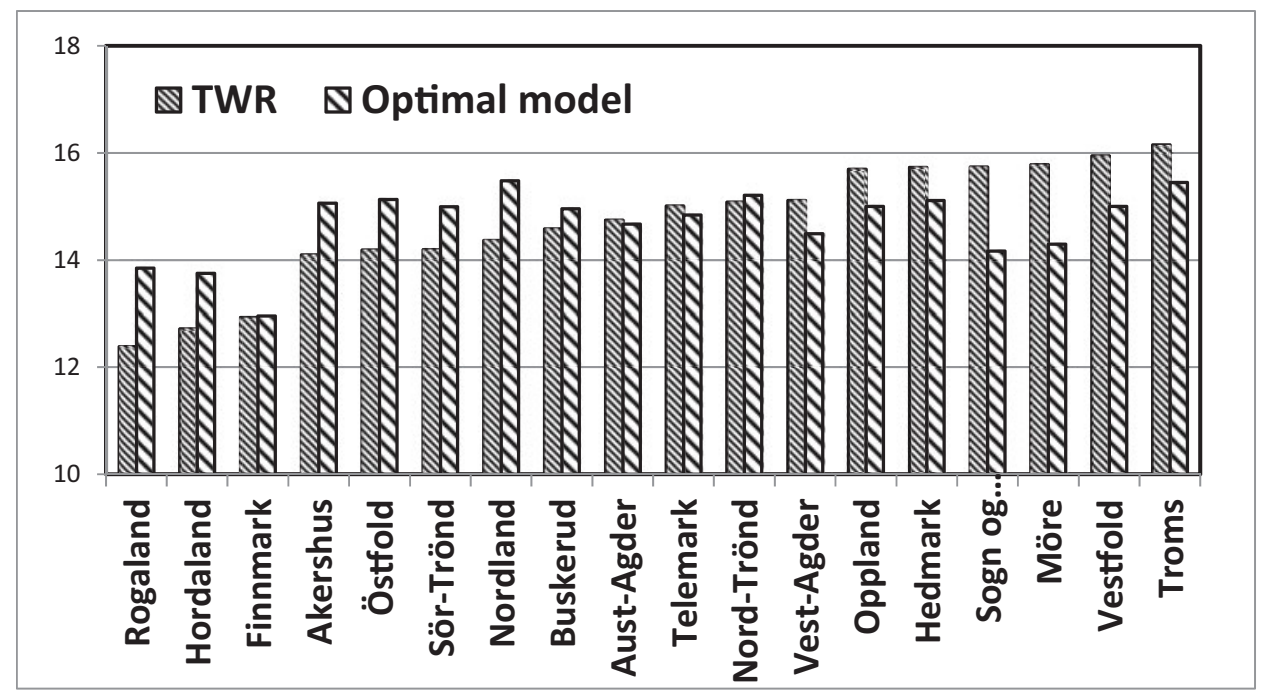

\section{FIGURE 4}

Observed and estimated regional TWRs. Note: The counties are ordered according to increasing observed TWRs. The estimated TWRs are based on model (5). The low goodness of fit discussed in the text can be observed in this figure.

show higher TWR levels, but also strong discrepancies from the model (cf. Figure 3). Therefore, our findings corroborate the weak results observed in the spatial modeling.

The low regional variation of the TWRs for 1960 1988 supports the finding that the regional TWRs for Sweden converged during the period from 1750 to 1960 towards a common low level (Fellman \& Eriksson, 2005b).

Comparisons of the multicollinearity in this Norwegian study and in the study of Sweden presented in Fellman and Eriksson (2009) show that the multicollinearity is markedly stronger in Norway than in Sweden. This is obviously a result of the fact that the two countries are almost of the same length, but Norway is much slimmer than Sweden. Furthermore, the spatial study of the TWR in Sweden yielded more successful spatial models than this study of the regional TWRs in Norway.

James (1985) observed a positive correlation coefficient (Spearman's) between the age-standardized TWR and latitude. He wondered whether this association of photoperiodicity with latitude is relevant. As alternative factors, he suggested diet (milk consumption) and birth weight. Bulmer (1970) has speculated that geographical variation in dizygotic TWRs in Europe may have some genetic basis. However, James (1985) stated that there is no reason to suppose that genetic clines in the Old World have been duplicated in the New World and the fact that the latitudinal variation in DZ twinning and birth weight are similar in Europe and the United States suggests an environmental rather than a genetic cause.

\section{Acknowledgments}

This work was supported by grants from The Finnish Society of Sciences and Letters and the Magnus Ehrnrooth Foundation.

\section{References}

Bulmer, M. G. (1970). The biology of the twinning in man. London: Oxford University Press.

Casella, G. (1980). Minimax ridge regression estimation. Annals of Statistics, 8, 1036-1056.

Eriksson, A. W. (1964). Pituitary gonadotrophin and dizygotic twinning. Lancet, 2, 1298-1299.

Eriksson, A. W. (1973). Human twinning in and around the Åland Islands. Commentationes Biologicae, 64, 1-159.

Eriksson, A. W., Abbott, C., Kostense, P. J., \& Fellman, J. O. (1993). Secular changes of twinning rates in Nordic populations. In E. Iregren and R. Liljekvist (Eds.), Populations of the Nordic countries Human population biology from the present to the Mesolithic: Proceedings of the Second Seminar of Nordic Physical Anthropology Lund 1990 (Report Series No. 46, pp. 113-135). Lund, Sweden: University of Lund, Institute of Archeology.

Eriksson, A. W., \& Fellman, J. (1999). Seasonal variations of twin maternities in Denmark: Secular and regional differences. Perspectives in Human Biology, 4, 213-221.

Eriksson, A. W., \& Fellman, J. (2004). Demographic analysis of the variation in the rates of multiple maternities in Sweden since 1751. Human Biology, 76, 343-359.

Fellman, J. O., \& Eriksson, A. W. (1990). Standardization of the twinning rate. Human Biology, 62, 803-816. 
Fellman, J. (1981). Leskinen's preliminary orthogonalizing ridge estimator and a new measure of multicollinearity (Working Papers Swedish School of Economics and Business Administration). Helsinki: Helsingfors.

Fellman, J. (2015). Temporal variation in rates of multiple maternities in Denmark, 1850-2012. Twin Research and $\mathrm{Hu}$ man Genetics, 18, 406-409.

Fellman, J. (2016). Historic demography of Iceland. British Journal of Medicine \& Medical Research, 2, 1-13.

Fellman, J., \& Eriksson, A. W. (1999). Secular changes in the seasonal patterns of births in Nordic countries. Perspectives in Human Biology, 4, 203-212.

Fellman, J., \& Eriksson, A. W. (2003). Temporal differences in the regional twinning rates in Sweden after 1750. Twin Research, 6, 183-191.

Fellman, J., \& Eriksson, A. W. (2004). Association between the rates of multiple maternities. Twin Research, 7, 387-397.

Fellman, J., \& Eriksson, A. W. (2005a). Variations in the maternal-age effect on twinning rates: The nordic experience. Twin Research and Human Genetics, 8, 515-523.

Fellman, J., \& Eriksson, A. W. (2005b). The convergence of the regional twinning rates in Sweden, 1751-1960. Twin Research and Human Genetics, 8, 163-172.
Fellman, J., \& Eriksson, A. W. (2009). Spatial variation in the twinning rate in Sweden, 1751-1850. Twin Research and Human Genetics, 12, 583-590.

James, W. H. (1985). Dizygotic twinning, birth weight and latitude. Annals of Human Biology, 12, 441-447.

Lawless, J. F. (1978). Ridge and related estimation procedures: Theory and practice. Communications in Statistics - Theory and Methods, A7, 139-164.

Mahajan, V., Jain, A. K., \& Bergier, M. (1977). Parameter estimation in marketing models in presence of multicollinearity: An application of ridge regression. Journal of Marketing Research, 14, 586-591.

Thisted, R. A. (1980). Comment on Smith and Campbell (1980). Journal of American Statistical Association, 75, 8186.

Vinod, H. D. (1976). Application of new ridge regression methods to a study of Bell system scale economics. Journal of American Statistical Association, 71, 835-841.

Wichern, D. W., \& Churchill, G. A. (1978). A comparison of ridge estimators. Technometrics, 20, 301-311. 